\title{
Úlceras orales recurrentes: Características clínicas y diagnóstico diferencial
}

Paola Toche P., Jessica Salinas L., M. Antonieta Guzmán M., Alejandro Afani S. y Nicole Jadue A.

\section{Recurrent oral ulcer: clinical characteristic and differential diagnosis}

Recurrent aphthous stomatitis (RAS), are common inflammatory lesions of the oral mucous, usually round or ovoid, circumscribed by an erytematous haloes with a yellow-grey floor and mostly painful. The RAS has reach an incidence about $20 \%$ in general population, present on any aged group, especially adolescents and young adults. Etiopathogenesis of RAS is not entirely understood. Some factors involved include immune system anomalies, infections, nutritional deficiency, mucous traumatism, food or contact allergy, autoimmunity illness and cancer; together with psychiatric, genetic and environment agents. In this article, main clinical features, etiology related factors, differential diagnosis and initial study of patients consulting for RAS are presented.

Key words: Oral ulcers, aphthous stomatitis, pathogenesis.

Palabras claves: Úlceras orales, estomatitis aftosa, patogénesis.

\section{Introducción}

$\mathbf{L}$ as úlceras orales son lesiones inflamatorias de la mucosa bucal que poseen múltiples etiologías. Pueden manifestarse de manera aguda, crónica o recurrente, en cuyo caso alcanzan una frecuencia aproximada de $20 \%$ en la población general ${ }^{1}$. Las úlceras orales recurrentes (UOR) comienzan en la niñez pero afectan, principalmente, a adolescentes y adultos jóvenes, deteriorando de manera importante la calidad de vida. En este artículo se discuten las causas más frecuentes y el enfrentamiento inicial de las UOR.

\section{Características clínicas}

Las UOR se caracterizan por ser dolorosas, ovaladas y circunscritas por un halo eritematoso con un fondo amarillo-grisáceo ${ }^{2}$. Se clasifican en:

- Úlceras menores. Son pequeñas (diámetro $<1 \mathrm{~cm}$ ), afectan entre 75 y $85 \%$ de los pacientes con UOR, curan en 7 a 14 días y recurren dos a cuatro veces por año. Estas lesiones afectan, generalmente, el epitelio no queratinizado (mucosa labial, bucal y piso de la boca; rara vez afectan a las encías, el paladar y dorso de la lengua) (Figura 1).

- Úlceras mayores. Caracterizan por tener un diámetro mayor a $1 \mathrm{~cm}$; afectan a $15 \%$ de los pacientes con UOR, son más profundas y dolorosas que las úlceras menores, curan más lentamente (en 10 a 30 días) y pueden dejar cicatriz. Afectan de preferencia a los labios y el paladar blando; sin embargo, pueden comprometer cualquier otro sitio (Figura 2).

- Úlceras herpetiformes: Se presentan como múltiples lesiones papulo-vesiculares agrupadas, con diámetro menor a $3 \mathrm{~mm}$, y pueden coalescer. $\mathrm{Su}$ nombre se debe a su aspecto semiológico, sin estar relacionadas con una etiología viral. Estas lesiones curan en siete a 30 días, pudiendo dejar cicatriz y constituyen el $10 \%$ de las UOR.

Otras clasificaciones de ulceras orales las dividen en úlceras agudas (hasta seis semanas de duración) y crónicas (más de 6 semanas) y según su número, en solitarias y múltiples ${ }^{3}$. Cabe destacar que las úlceras crónicas pueden ser indoloras y deberían ser siempre biopsiadas, con el fin de descartar una etiología maligna o autoinmune.

\section{Etiología}

Se han mencionado numerosas causas y factores asociados que podrían jugar un rol en la génesis de estas lesiones. Entre ellas, destacan alteraciones inmunológicas, infecciones, déficit nutricionales, trau-

\section{Hospital Clínico Universidad de Chile}

Departamento de Inmunología

Recibido: 13 de junio de 2006 Aceptado: 25 de enero de 2007

Correspondencia a:

Paola Toche Pinaud paolatoche@yahoo.com 
Figura 1. Se muestra un ejemplo de UOR menor, nótese su ubicación en el epitelio no queratinizado, su tamaño menor a $1 \mathrm{~cm}$ de diámetro, su borde eritematoso y fondo amarillo-grisáceo característico.

Figura 2. Se observa un ejemplo de UOR mayor caracterizada por su diámetro mayor a $1 \mathrm{~cm}$, profundidad y ubicación en la mucosa labial.
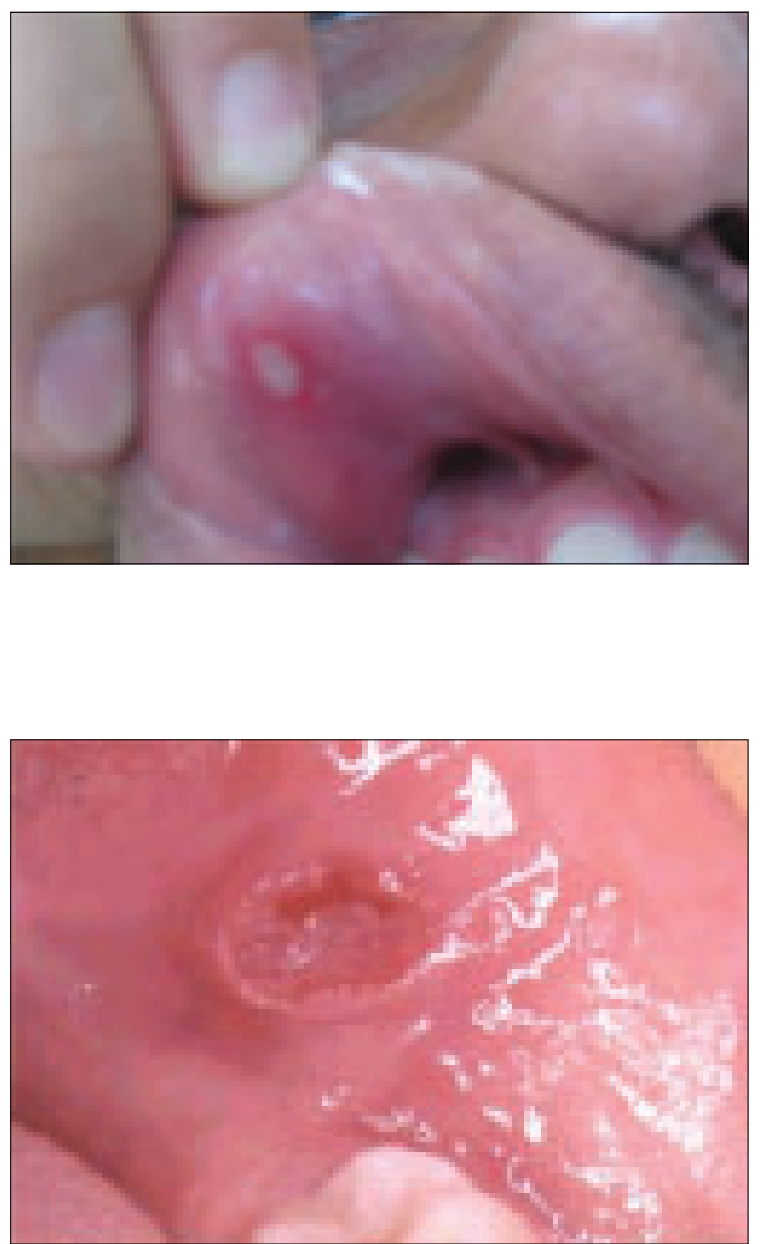

matismo repetitivo de la mucosa oral, alergia alimentaria y de contacto, enfermedades autoinmunes y neoplasias, junto con factores psiquiátricos $\mathrm{y}$ genéticos ${ }^{2,4,5}$.

Es fundamental estudiar las causas subyacentes de las UOR, especialmente de las úlceras mayores que son, precisamente, las que llevan a los pacientes a consultar, con el fin de tratar la patología de base y no sólo brindar un tratamiento sintomático de estas lesiones.

\section{Aspectos inmunológicos infecciosos de las UOR}

Ambos aspectos han sido ampliamente estudiados en la etiopatogenia de las UOR.

Inmunidad. Dentro de las alteraciones inmunológicas, existe una clara evidencia del compromiso de la inmunidad celular. Algunos estudios muestran una disminución del recuento de linfocitos CD4+ en sangre periférica, con una disminución de linfocitos TCD4+CD45+RA (células naive) y un aumento de los linfocitos CD4+ CD25+RO (células de memoria) lo que indicaría una activación linfocitaria ${ }^{2}$. Además, se ha observado que los pacientes infectados con VIH, con relación $\mathrm{CD} 4+/ \mathrm{CD} 8+$ invertida, presentan una prevalencia de UOR mayor que la población general ${ }^{4}$.

También se ha demostrado un importante rol del factor de necrosis tumoral alfa (FNT $\alpha)$ sobre las células endoteliales y la quimiotaxis de neutrófilos en las $\mathrm{UOR}^{6}$. Con respecto a la inmunidad humoral, se describe, en general, con niveles de inmunoglobulinas normales, sin otras alteraciones específicas asociadas.

Infecciones. Con respecto al rol de algunos microorganismos, inicialmente se postuló que la infección por algunos estreptococos orales, (Streptococcus sanguis, Streptococcus grupo viridans), se relacionaba con las $\mathrm{UOR}^{7-9}$, lo cual fue refutado en trabajos posteriores ${ }^{10}$. Nuevos estudios han sugerido que podría existir reactividad cruzada entre algunas proteínas de shock térmico de Mycobacterium tuberculosis (también presentes en $S$. sanguis) y la mucosa oral, la que podría ser dañada por linfocitos $\mathrm{T}$ dirigidos contra estas proteínas ${ }^{11}$.

Es importante destacar que algunos virus pueden producir ulceras orales, generalmente de forma aguda, en un contexto infeccioso claro. Se ubican en la mucosa queratinizada y una característica clave es que son precedidas por vesículas en etapas iniciales, las que, al romperse, evolucionan a la conformación de úlceras orales úlceras orales que no son recurrentes, a diferencia de aquellas causadas por el virus herpes simplex tipo 1 (VHS-1) que sí tienden a la recurrencia (Tabla 1).

La evidencia actual no ha logrado demostrar una 
asociación sólida entre VHS o fragmentos de él y las UOR. Sin embargo, algunos trabajos describen la presencia de ADN de virus herpes tipo 8 y virus varicela $z^{2}$ ster $^{12}$ en UOR de pacientes con deterioro de la inmunidad celular (linfoma de Hodgkin, tratamientos inmunosupresores o infección por VIH), y ADN de citomegalovirus (CMV) en pacientes inmunocompetentes $^{2,6}$.

El VHS-1 puede producir úlceras recurrentes en la fase post-vesiculosa, que merecen ser discutidas por la frecuente confusión que causan con las UOR. Las úlceras causadas por VHS-1 se ubican en la mucosa queratinizada o parcialmente queratinizada (comisura labial, paladar duro, mucosa gingival peridental), tienden a recurrir en el mismo sitio y pueden ser precipitadas por factores como estrés y trauma, al igual que las $\mathrm{UOR}^{12,13}$.

Otras causas de úlceras orales de origen infeccioso, generalmente crónicas, son la sífilis primaria y terciaria y la tuberculosis, que puede presentarse como úlcera única o múltiple ${ }^{14}$.

\section{Otros factores etiológicos en las úlceras orales recurrentes}

Trauma. Es un factor que se asocia frecuentemente a las UOR y generalmente su origen es mecánico o accidental. Pueden ser producidos por una mala oclusión dental, en cuyo caso las úlceras tienden al recurrir en el mismo sitio ${ }^{15}$. También se producen por el cepillo de dientes, inyecciones, e incluso, por alimentos de borde cortante ${ }^{16,17}$. El rol del tabaco es controversial ya que pese a ser una forma de agresión de la mucosa oral, tendría un rol protector. Los pacientes fumadores presentan UOR menos severas y con menor frecuencia que los no fumadores, hallazgo que sería explicado por una mayor queratinización del epitelio producido por la nicotina ${ }^{18}$.

Déficit nutricionales. La carencia de fierro, ácido fólico o vitaminas del complejo $\mathrm{B}_{12-} \mathrm{B}_{6} \mathrm{y}$ oligoelementos tales como zinc, se han relacionado de manera variable con las $\mathrm{UOR}^{10,16}$. El tratamiento de reemplazo de estas deficiencias mejora las UOR en algunos casos.

Enfermedades autoinmunes. Las UOR pueden ser la primera manifestación de enfermedades sistémicas tales como la enfermedad de Behcet, la que se acompaña de úlceras genitales, además de alteraciones oculares (uveítis), neurológicas y cutáneas (pseudofoliculitis o nódulos acneiformes) ${ }^{19-21}$. El lupus eritematoso sistémico también puede causar UOR, generalmente acompañadas de manifestaciones articulares, cutáneas y sistémicas.
Enfermedades gastrointestinales: La enfermedad celíaca, cuya presentación clínica es cada vez más atípica, puede manifestarse con UOR en aproximadamente $4 \%$ de los casos; en la mayoría de éstos, las úlceras remiten con dietas libres de gluten ${ }^{22}$. Algunos trabajos muestran que las enfermedades inflamatorias intestinales como la enfermedad de Crohn pueden manifestarse con UOR hasta en $10 \%$ de los pacientes $^{23}$.

Enfermedades cutáneas. Las enfermedades bulosas (pénfigo y penfigoide) pueden acompañarse de UOR. El diagnóstico puede resultar difícil en aquellos casos sin lesiones cutáneas y en los que se visualiza la úlcera una vez que la bula mucosa se ha roto $^{24}$. En estos casos, la biopsia con inmunofluorescencia es un aporte fundamental en el diagnóstico. Otra causa importante a considerar entre las afecciones dermatológicas es el liquen plano.

Medicamentos. Los agentes citotóxicos, al igual que la radioterapia, afectan a las células en activa división como sucede con la mucosa oral, produciendo en algunos pacientes úlceras orales. Otros fármacos que se han relacionado con las úlceras son el ácido niflumico, captopril, piroxicam y fenobarbital, entre $\operatorname{otros}^{25,26}$.

Alteraciones hematológicas. La neutropenia (benigna o cíclica), anemia y los síndromes mielodisplásicos también se han asociado a UOR ${ }^{25}$. Entre otras afecciones que se caracterizan por la presencia de UOR, se encuentra el síndrome de PFAPA (del inglés: periodic fever, aphtae, pharyngitis, adenopathies), en el que las úlceras están presentes en cerca de $65 \%$ de los pacientes y se caracterizan por su número variable, tamaño pequeño $(<0,5 \mathrm{~mm})$, presentación no agrupada y superficial, de forma que aparecen en concomitancia con la fiebre y curan sin dejar cicatriz, en 5 a 10 días $^{27-29}$. El síndrome de Sweet puede presentar UOR acompañadas de fiebre, leucocitosis neutrofílica y nódulos o placas eritematosas en la piel, pudiendo asociarse neoplasias subyacentes como leucemias ${ }^{26}$.

Alergia. La hipersensibilidad a antígenos alimentarios o de contacto (presentes en pastas dentífricas o materiales dentales) ha sido poco estudiada y escasamente documentada ${ }^{16,30}$. El rol real que juega la alergia en las UOR aún no ha sido establecido.

Estrés. Se ha identificado este factor como un importante desencadenante de la aparición de UOR. Se ha sugerido algún beneficio con el uso de antidepresivos en algunos pacientes con $\mathrm{UOR}^{31}$.

En suma, las causas de UOR son variadas, la historia clínica y el examen físico deben orientar a un estudio inicial que se resume en la Tabla 2. 
Tabla 2. Etiologías y estudio inicial de las úlceras orales recurrentes

\begin{tabular}{ll} 
Patología & Orientación diagnóstica y estudio \\
\hline $\begin{array}{c}\text { Déficit nutricionales } \\
\text { Déficit de fierro, ácido fólico, } \\
\text { complejo } B_{12}, B_{6} \text { y zinc }\end{array}$ & $\begin{array}{l}\text { Nutrición desbalanceada } \\
\text { Hemograma } \\
\text { Determinación de niveles séricos de complejo B y zinc }\end{array}$
\end{tabular}

Enfermedades autoinmunes

Lupus eritematoso sistémico Síntomas articulares, cutáneos, oculares, neurológicos Enfermedad de Behcet ANA, ENA, C3 y C4. Test de Patergia

Trauma Evaluar oclusión dental

Enfermedades cutáneas

$\begin{array}{ll}\text { Pénfigo } & \text { Lesiones bulosas en piel (no siempre presentes) } \\ \text { Penfigoide } & \text { Biopsia e inmunofluorescencia }\end{array}$

Liquen plano

Carcinoma escamoso

Medicamentos

Ácido niflumico Antecedente de ingestión de fármacos

Captopril

Eventual realización de pruebas de parche

Piroxicam

Fenobarbital, entre otros

Enfermedades hematológicas
Neutropenia cíclica
S. anémicos
S. mielodisplásicos
S. de Sweet
S. de PFAPA

Enfermedades gastrointestinales

Enfermedad celíaca

Enfermedad de Crohn

Dolor abdominal, diarrea, anemia, talla baja

Anticuerpos anti endomisio, anti giadina,

anti transglutaminasa

Estudio endoscópico y biopsia

Alergia

Alimentaria

Contactantes dentales

Sospecha de relación con determinados alimentos

Pruebas de parche para alimentos

Pruebas de parche para pastas y materiales dentales

PFAPA: fiebre periódica, aftas, faringitis y adenitis

\section{Referencias}

1.- Scully C, Shotts R. ABC of oral health. Mouth ulcers and other causes of orofacial soreness and pain. Br Med J 2000; 321: 162-5.

2.- Porter S R, Scully C, Pedersen A. Recurrent aphthous stomatitis. Crit Med Oral Biol Med 1998; 9: 306-21.

3.- Schneider L C, Schneider A E. Diagnosis of oral ulcers. Mt Sinai J Med 1998; 65: 383-7.

4.- Shashy R G, Ridley M B. Aphthous ulcers: a difficult clinical entity. Am J Otolaryngol 2000; 21: 389-93.

5.- Porter S R, Hegarty A, Kaliakatsou F, Hodgson T A, Scully C. Recurrent aphthous stomatitis. Clin Dermatol 2000; 18: $569-78$.

6.- Natah S S, Hayrinen-Immonen R, Hietanen J, Malmstrom M, Konttinnen Y T. Immunolocalization of tumor necrosis factor alfa expressing cells in recurrent aphthous ulcers lesions (RAU). J Oral Pathol Med 2000; 29: 19-25.

7.- Porter S R, Scully C, Pedersen A. Recurrent aphthous stomatitis. Crit Rev Oral Biol Med 1998; 9: 306-21.

8.- Vincent S D, Lilly G E. Clinical, historic, and therapeutic features of aphthous stomatitis. Literature review and open clinical trial employing steroids. Oral Surg Oral Med Oral Pathol 1992; 74: 79-86.

\section{Conclusiones}

Las úlceras orales recurrentes son lesiones frecuentes y clínicamente muy similares entre sí. El diagnóstico etiológico se basa en la presencia de síntomas y signos asociados, junto con el estudio de laboratorio. La realización de biopsia es necesaria frente a sospecha de patologías neoplásicas o autoinmunes.

\section{Resumen}

Las úlceras orales recurrentes (UOR), son lesiones inflamatorias frecuentes de la mucosa oral. Generalmente, son redondas u ovaladas, rodeadas por un halo eritematoso de fondo amarillo grisáceo y dolorosas en su mayoría. Las UOR alcanzan una frecuencia de hasta $20 \%$ en la población general, afectando a cualquier grupo etario, en especial a adolescentes y adultos jóvenes. La etiopatogenia de las UOR no está completamente dilucidada; algunos factores involucrados incluyen alteraciones inmunológicas, infecciones, déficit nutricional, traumas de la mucosa, alergia alimentaria y de contacto, enfermedades autoinmunes y neoplasias; junto con factores psiquiátricos, genéticos y medioambientales. En el presente artículo se discuten las características clínicas, factores etiológicos, diagnósticos diferenciales y el estudio de las UOR. 
infections and cytokines associated with recurrent aphthous ulceration. Microbes Infect 2005; 7: 635-44.

13.- Eisen D. The clinical characteristics of intraoral herpes simplex virus infection in 52 immunocompetent patients. Oral Surg Oral Med Oral Pathol Oral Radiol Endod 1998; 86: 432-7.

14.- Woo S B, Lee S F. Oral recrudescent herpes simplex virus infection. Oral Surg Oral Med Oral Pathol Oral Radiol Endod 1997; 83: 239-43.

15.- Szpirglas H. Diagnosis of mouth ulcers. Rev Prat 2002; 52: 375-9.

16.- Rees T D, Binnie W H. Recurrent aphthous stomatitis. Dermatol Clin 1996; 14: 243-56.

17.- Ship J A. Recurrent aphthous stomatitis. An update. Oral Surg Oral Med Oral Path Oral Radiol Endod 1996; 81: 141-7. Review.

18.- Axell T, Henricsson V. Association between recurrent aphthous ulcers and tobacco habits. Scand J Dent Res 1985; 93: 239-42.

19.- Rogers R S 3rd. Recurrent aphthous stomatitis in the diagnose of Behcet's disease. Yonsei Med J 1997; 38: 370-9. Review.

20.- Areny R, Saavedra J. Enfermedad de Behcet: Experiencia en el Departamento de Reumatología. Hospital San Juan de Dios. Reumatología 2003; 19: 150-2.

21.- López de Maturana D, Amaro P, Balestrini C, Segovia L. Manifestaciones clínicas en 5 casos de enfermedad de Behcet. Rev Méd Chile 2002; 130: 551-6.

22.- Schuppan D. Current concept of celiac disease pathogenesis. Gastroenterology 2000; 119: 234-42. Review.

23.- Feighery C. Fortnightly review: Coeliac disease. Br Med J 1999; 319: 236-9.

24.- Scully C, Challacombe S J. Pemphigus vulgaris: update on etiopathogenesis, oral manifestations, and management. Crit Rev Oral Biol Med 2002; 13: 397-408.

25.- Scully C, Gorsky M, Lozada-Nur F. The diagnosis and management of recurrent aphthous stomatitis: a consensus approach.
J Am Dent Assoc 2003; 134: 200-7. 26.- Agbo-Godeau S. Apthous stomatitis. Rev Prat 2002; 52: 380-4.

27.- Long S. Syndrome of Periodic Fever Aphthous stomatitis, Pharyngitis and Adenitis (PFAPA) What it isn't. What is it? J Pediatr 1999; 135: 1-5.

28.- Padeh S, Brezniak N, Zemer D, Pras E, Livneh A, Langevitz P, et al. Periodic fever, aphthous stomatitis, pharyngitis and adenopathy syndrome: clinical characteristics and outcome. J Pediatric 1999; 135: 98-101.

29.- Castro M, Arredondo A, Talensnik E. Síndrome de fiebre periódica, faringitis, aftas y adenitis cervical. Rev Chil Pediatr 2003; 74: 294-8.

30.- Woo S, Sonis S. Recurrent aphthous ulcers: A review of diagnosis and treatment. J Am Dent Assoc 1996; 127: 1202-13. Review.

31.- Pedersen A. Psychologic stress and recurrent aphthous ulceration. J Oral Pathol Med 1989; 18: 119-22. 